\title{
THE RAT PACK
}

\section{Studying primates is the only way to understand human cognition - or so neuroscientists thought. But there may be much to learn from rats and mice, finds Alison Abbott.}

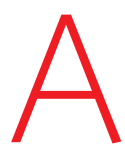
nne Churchland had little time for rats. In the course of 13 years' work on decision-making in monkeys, she had never questioned that primate studies were the only way to understand the neurobiology of human cognition. Her work in the lab of Michael Shadlen at the University of Washington, Seattle, had monkeys watch moving dots flitting about on a screen until the animals indicated, with a flick of their eyes, the direction in which most of the dots were going. She recorded from single brain neurons as the monkeys slowly made sense of this 'fuzzy' information - the sort of sophisticated experiment that she did not think was possible in rodents.

"I didn't think rats would have the right sorts of brains to contemplate accumulating evidence," says Churchland. And with poor eyesight, and heads that bob around, "I didn't imagine they would be able to convey to us any decision they might be silently making".

All that changed a year ago, when Churchland visited Cold Spring Harbor Laboratory in New York. Working with scientists there, she saw that rats could also learn to gather 'fuzzy' sensory information - in this case to decide whether the frequency of a rapid sequence of tones was mostly high or low. And they could convey their decision with a poke of the nose.

Churchland was not alone in her earlier scepticism. Neurophysiological research into higher cognitive functions such as decision-making, attention, working memory - even risk-taking - have traditionally been carried out on non-human primates. That seemed an obvious choice, given the closeness of their brain anatomy to that of humans, the sophistication and breadth of their behaviour and their ability to reliably report to experimenters much of what is going on in their minds through eye, hand or other movements. But primate work comes with major downsides: the animals are so expensive, and their use so highly regulated, that a research paper typically relies on data from just a couple of precious animals, which have been used for multiple experiments over their lifetime. This raises concerns that observations could be unique to those animals, rather than a general property of the primate brain.

Mice and rats, by contrast, can be studied in the tens or hundreds. But with brains a fraction of the size of those of humans or non-human primates - and no prefrontal cortex, the highly-evolved brain area where 'higher' cognition is thought to take place - neuroscientists assumed that rodents were simply incapable of learning complex behavioural paradigms.

That scepticism is dissolving, thanks in large part to a 'rodent cognition movement' started by a small group of researchers at Cold Spring Harbor almost a decade ago and now spreading far beyond its grounds. Using carefully designed tasks, these researchers have shown that rodents can undertake some types of complex cognitive behaviour just like experimental primates, and just like humans.

"Primate used to be the only game in town," says Zachary Mainen, now at the Champalimaud Neuroscience Programme in Lisbon, Portugal, but one of the founders of the rodent movement when he was at Cold Spring Harbor. "Now we are starting to appear as a small force in cognition meetings."

\section{Evolutionary similarities}

Mainen joined Cold Spring Harbor Laboratory in 1999, the same year as his colleague Tony Zador. Both wanted to move beyond their backgrounds in computational neuroscience and cellular neurophysiology, and find out how electrical activity in neurons - such as that stimulated by sensory input - related to behaviours such as decision-making. They thought that these components of behaviour "would likely be evolutionarily similar across mammals", says Mainen. And rats, they thought, would move the field forwards faster than primates, particularly given the greater availability of tools for manipulating rodent genes.

Zador sees the choice of primates for $\operatorname{cog}$ nition experiments as a "historical accident", naturally evolving from research begun in the 1960 s to understand how vision was processed in the brain. "Using primates made complete sense, because vision is so highly specialized in primates for functions such as face recognition," he says. Then, in the 1980s, some primate-research groups went on to ask how visual information couples to motor output; having seen an object, what happens in an animal's brain as it decides whether to reach for it? The interesting questions were
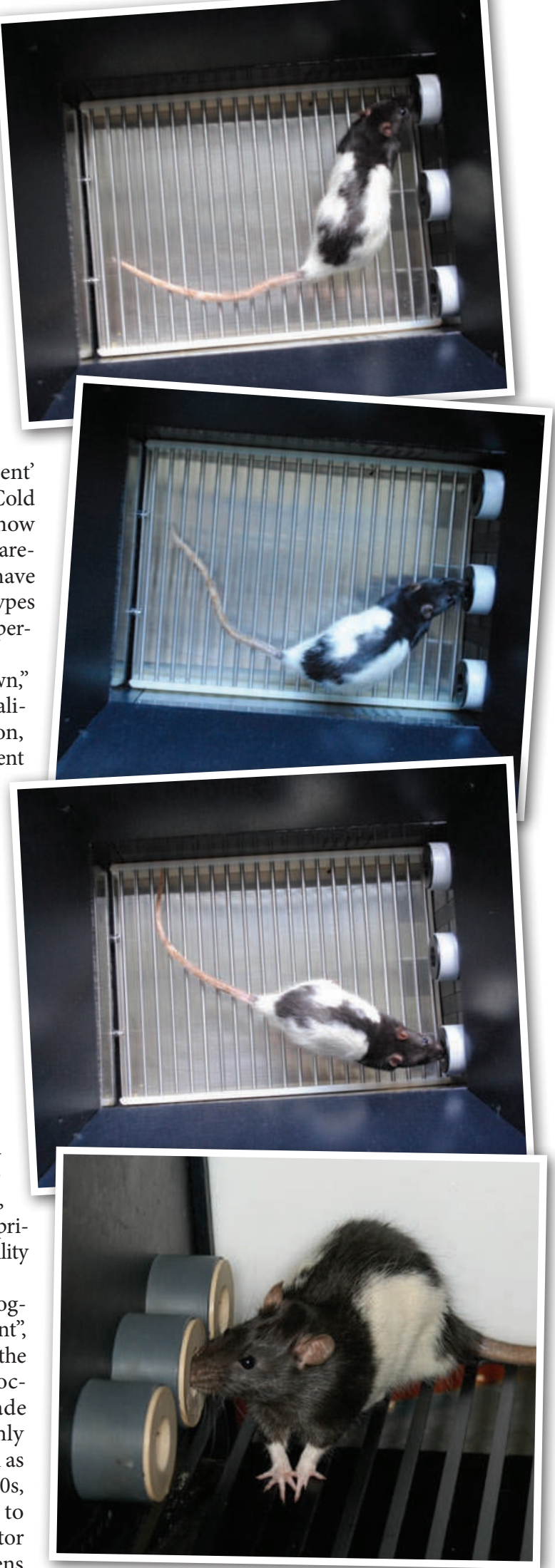

A rat indicates a decision by poking its nose through a 'port' when it has discriminated between two odours. 
now about cognition. "At this point primates offered no unique advantage, because the tasks that the researchers were asking monkeys to do were so simple," says Zador.

To study these tasks in rats, Zador and Mainen had to decide what sensory system to use, and establish a behavioural read-out. Rodents primarily rely on senses other than vision, such as hearing and smell, to guide their behaviour. So Zador began to look at how rats processed auditory information; Mainen focused on odours.

It took a few years, and a lot of trial and error. But by 2003 Mainen had published his first paper ${ }^{1}$ showing that rats could be trained to reliably repeat behaviour, discriminating between similar smells after a single whiff.
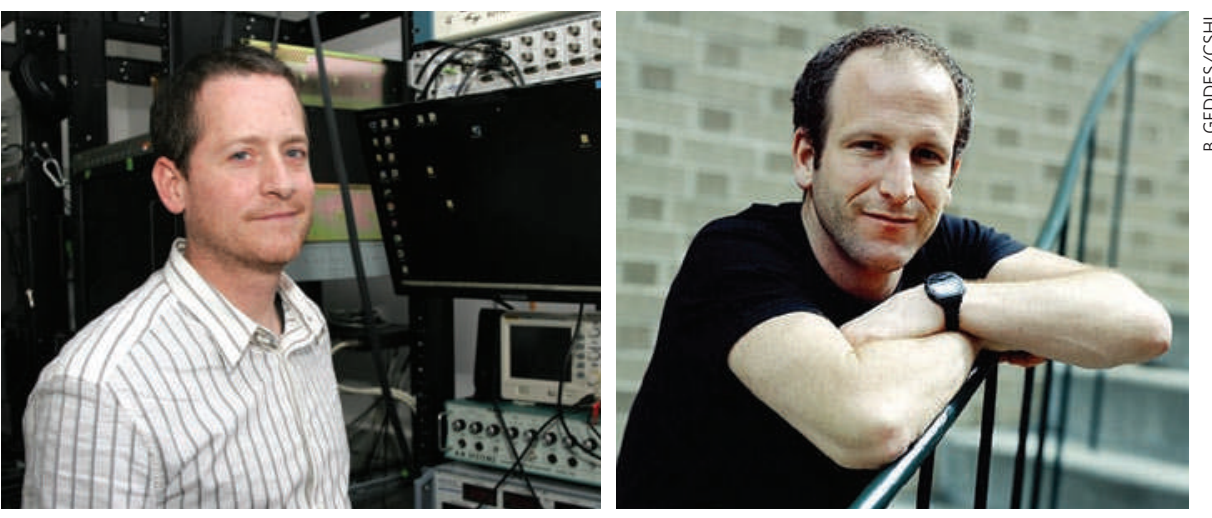

Researchers including Zachary Mainen (left) and Tony Zador founded a 'rodent cognition movement'. More to the point, he showed that they could indicate their detection of an odour by poking their noses through a 'port' in the cage wall.

That paper was an eye-opener for Carlos Brody, a computational neuroscientist at Cold Spring Harbor. "I had theories that I would have liked to test in a primate lab, but this paper showed that you could do equally rigorous work with rats," he says. Brody joined forces with Mainen and Zador and the three of them persuaded the laboratory to set up the Center for Neural Mechanisms of Cognition in 2006, devoted to rodent work.

In 2008, Mainen published a second watershed paper, using electrophysiology to show how rats make everyday decisions on the basis of fuzzy evidence ${ }^{2}$. This time he trained rats to distinguish between two odours delivered through the central port of a row of three. If a mixture had more of odour one, the rat had to poke its nose through the left-hand port; if it had more of odour two, it had to select the right-hand one. The decision became very difficult when the mixtures contained nearly equal parts of the two odours, but if the rats decided correctly, and waited long enough at the correct

"The rat brain shares
fome of the most
principles with those
of humans."

activity can be switched on or off with flashes of laser light ${ }^{3}$, allowing the role of neurons in a behaviour circuit to be dissected. Right now these systems work best in mice. But because most behavioural studies have been carried out using rats, cognitive scientists have mostly chosen to start on rats in the hope that the techniques will be quickly transferred. Zador says it's still not clear whether the smallerbrained mouse is capable of the behaviours in which the field is interested.

\section{Rodent logic}

Churchland is so taken with the experimental possibilities afforded by rodents that she is staking her career on them. This summer she is moving to Cold Spring Harbor, where she will establish her own lab to study rat decision-making. Looking back, she wonders why she doubted rats' cognitive abilities so much. "They also have to make decisions in the wild in order to survive, and would obviously have to accumulate and sift evidence to do so." She wants to explore why some rats choose a strategy of decisionmaking that sacrifices accuracy for speed. "With higher numbers, we can start to look port, they received a drink reward there. The more confident rats were about their decision, Mainen found, the longer they were prepared to wait. And when he took recordings from single neurons in the orbital frontal cortex, a brain area involved in decision-making, he found patterns of electrical activity that correlated with the rats' conviction. "It hadn't been clear whether the rat brain was going to be up to the task of estimating confidence in decisions," says Mainen. "But we showed it was, and at least in this sort of task, rats are as good as monkeys as subjects."

In certain ways, rodents are better. In the past few years the development of 'optogenetic' tools has allowed rodent researchers to engineer particular neurons so that their at individual differences," she says.

Other committed primate researchers, such as Daeyeol Lee, a neuroeconomist at Yale University School of Medicine in Connecticut, are also exploring the use of rodents. Lee has been working on primate decision-making for 15 years. "The rat brain shares some of the most fundamental design principles with those of humans and other primates, such as connectivity between the cortex and some sub-cortical areas," he says. "Rats may be able to teach us a lot." And behavioural researchers are working to see how far they can go with rodents, developing new paradigms in rats that might even mimic some classic human psychology tests, including a version of the Iowa gambling task, which probes the ability to make appropriate decisions in the face of stacked odds ${ }^{4}$. Researchers have also claimed that a paradigm based on the prisoner's dilemma, which explores why people might not cooperate even when it is in their best interests, shows that rats can understand the complex pay-offs that cooperation entails ${ }^{5}$.

Some primate researchers, though, remain unconvinced. "It is good to develop rodent models and see what they are capable of," says Shadlen. "But it still isn't clear to me that rodents do any serious deliberating in decision-making." And Daniel Salzman at Columbia University in New York says that the differences, rather than the similarities, in brain anatomy and circuitry are going to be decisive, such as the smaller rodent frontal cortex. Rodent researchers "are quickly going to run up against a wall," he predicts.

Still, few on either side of the species divide care to be too categorical. Rodent-cognition researchers have presented enough new data at meetings to discourage dogmatism from primate loyalists. And rodent proponents emphasize that primates will always be required to reality-check the theories about cognition spawned by rodent research.

"Primates are going to be capable of some cognitive processes that rats are simply not capable of," says Brody, who thinks both types of research should run in parallel. "But the jury is still very much out in terms of where the capability border lies, and we think it is worth finding out."

Alison Abbott is Nature's senior European correspondent.

\footnotetext{
. Uchida, N. \& Mainen, Z. F. Nature Neurosci. 6 1224-1229 (2003).

2. Kepecs, A., Uchida, N., Zariwala, H. A. \& Mainen, Z. F. Nature 455, 227-231 (2008)

3. Buchan, L. Nature 465, 26-28 (2010)

4. Zeeb, F. D., Robbins, T. W. \& Winstanley, C. A Neuropsychopharmacol 34, 2329-2343 (2009).

5. Viana, D. S., Gordo, I., Sucena, E. \& Moita, M. A. PLOS ONE 5, e8483 (2010).
}

See Editorial, page 267. 